\title{
The power of fluctuations
}

\author{
The 2021 Nobel Prize in Physics has been awarded to Syukuro Manabe, Klaus Hasselmann and Giorgio Parisi "for \\ groundbreaking contributions to our understanding of complex physical systems".
}

E ach year, on the morning of the first Tuesday in October, it is customary for one of the physics editors at Nature towers to suddenly remember the Nobel Prize is about to be announced. In turn, they fire off a message reminding their editorial colleagues, so they can put aside any notions of doing any work that morning. And then, inevitably, the question comes up: "so who's going to win it this time?"

And naturally, we haven't got a clue. There are, of course, preferences expressed and bets made - one or two of us will also claim not to be fans of the idea of giving so much credit to so few individuals at all - but whenever somebody says "what about complex systems?", it only results in a further question: "but who would they give that to?"

In awarding one half of the 2021 prize to Syukuro Manabe and Klaus Hasselmann "for the physical modelling of Earth's climate, quantifying variability and reliably predicting global warming" and the other half to Giorgio Parisi "for the discovery of the interplay of disorder and fluctuations in physical systems from atomic to planetary scales", the Nobel committee gave a compelling answer.

Fluctuations and the subtle mechanisms through which ordered phenomena emerge within disordered and chaotic systems play an essential and much under-appreciated role in determining properties and dynamics that become manifest on all length scales, from materials such as glass all the way to the climate.

An important skill for a scientist is to identify a correct minimal model that economically captures the essential phenomena under investigation. Syukuro Manabe's work in the 1960s exploring the interaction between radiation balance and the vertical transport of air masses in the atmosphere is a case in point: he first modelled this as a one-dimensional column, but in doing so he captured how increased levels of carbon dioxide in the atmosphere lead to increased temperatures at the surface of the Earth.

All models carry their limitations and pitfalls, and understanding these is essential for making a meaningful connection with empirical observation. For a system as complex and chaotic as the atmosphere, in which the weather can be notoriously unpredictable on a timescale of days, it is especially important to understand why

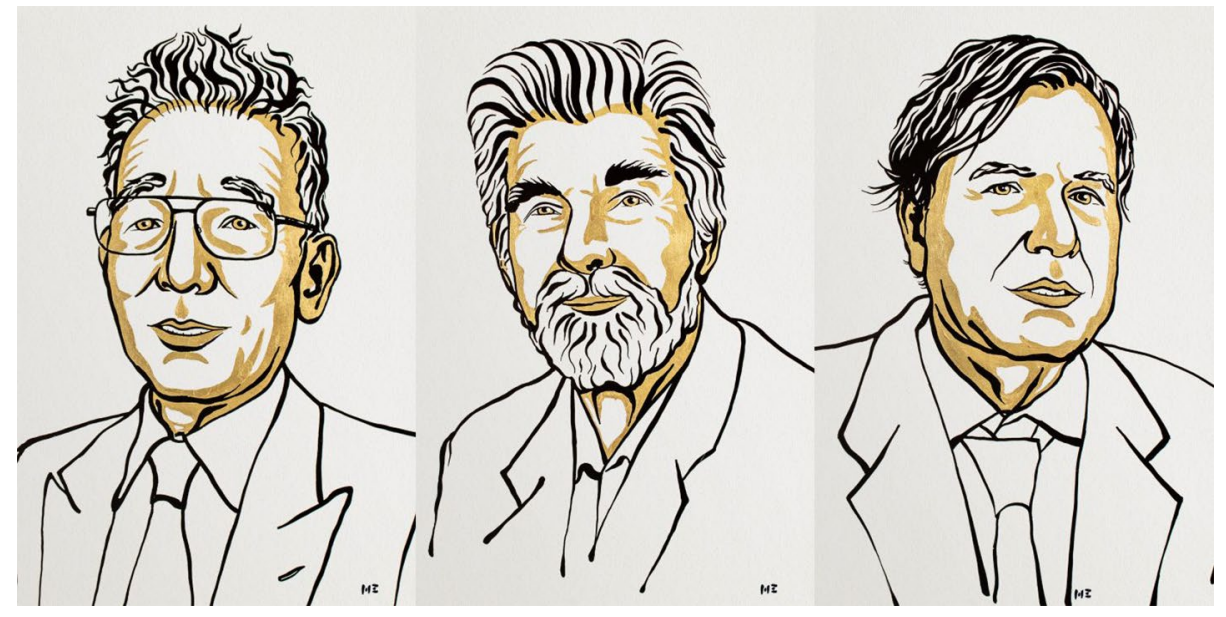

Syukuro Manabe, Klaus Hasselmann and Giorgio Parisi . Credit: (c) Nobel Media AB 2021 / Niklas Elmehed.

climate models can be considered reliable on the timescale of months and years.

Klaus Hasselmann's work provided some deep insights in this regard: by drawing an analogy with Brownian motion, he formulated a stochastic model that provides a handle on the climate's variability, and helps avoid the pitfalls of misinterpreting statistical quantities such as the variance and the mean in observational data. As a result, he was able to provide a framework that allows the separation of the signal (the large-scale dynamics of the climate) from the noise (the short-range changes in the weather), and furthermore, develop methods for identifying specific fingerprints of natural and human-induced effects in these data. These approaches have been used to prove that the increased temperature in the atmosphere is due to human emissions of carbon dioxide.

Stochastic models of the climate are built on foundational concepts of hydrodynamics, and share a deep connection with statistical mechanics. In a fundamental sense, both disciplines ultimately grapple with exploring hugely complex energy landscapes that arise from nonlinear effects due to disorder and fluctuations on one length scale, which make their consequences manifest at much larger ones. And what better example of this phenomenon than glass, a material that ages on geological time scales due to the subtle effects of impurities and disorder on the atomic scale.
Giorgio Parisi's work on spin glasses, in particular the replica symmetrybreaking solution he devised in order to calculate the partition function of these magnetic analogues of glass, is perhaps his most famous contribution. But in a career spanning many decades, his insights in a range of different areas in mathematics, neuroscience and machine learning are a testament of the power of his theoretical approach, and are among the most important contributions to the theory of complex systems.

There is a deep humility that lies at the root of the scientific approach espoused by scientists such as Manabe, Hasselmann and Parisi, which requires one to embrace uncertainty, variation and even doubt. Contrary to the perception that more is better, in order to understand a phenomenon it isn't necessary to construct a faithful representation of the entire physical system including as many of the fine details as possible. But it is essential to identify the fundamental ingredients that really matter to understand the problem, and appreciate their (at times, unintended) effects over vastly different time scales. When it comes to climate change, we must hope that we also find the collective humility to do something about it.

Published online: 8 November 2021 https://doi.org/10.1038/s41567-021-01420-y 